Daniela Parente de Mello Modiano

\title{
Um modelo de avaliação dos fatores sistêmicos determinantes do valor e custo de capital de uma empresa nos países desenvolvidos e nos emergentes
}

Dissertação de Mestrado (Opção profissional)

Dissertação apresentada como requisito parcial para obtenção do título de Mestre pelo Programa de PósGraduação em Administração da PUC-Rio.

Orientador: Luiz Felipe Jacques da Motta 


\title{
Pontificia Universidade Catollica DO RIO DE JANEIRO
}

Daniela Parente de Mello Modiano

\begin{abstract}
Um modelo de avaliação dos fatores sistêmicos determinantes do valor e custo de capital de uma empresa nos países desenvolvidos e nos emergentes
\end{abstract}

Dissertação apresentada como requisito parcial para obtenção do título de Mestre pelo Programa de PósGraduação em Administração da PUC-Rio. Aprovada pela Comissão Examinadora abaixo assinada.

Luiz Felipe Jacques da Motta

Orientador IAG-PUC

Antonio Carlos Figueiredo Pinto PUC-Rio

Marco Antonio Cunha de Oliveira FACC - UFRJ

Marcelo Cabus Klotzle PUC-Rio

Prof. João Pontes Nogueira Coordenador(a) Setorial do Centro de Ciências Sociais - PUC-Rio 
Todos os direitos reservados. É proibida a reprodução total ou parcial do trabalho sem autorização da universidade, da autora e do orientador.

\section{Daniela Parente de Mello Modiano}

Graduou-se em Engenharia Mecanica de Producao pela PUC-Rio em 2000. Trabalhou como analista financeiro em empresa de mineração e consultorias de finanças corporativas.

Ficha Catalográfica

\begin{tabular}{|c|}
\hline Modiano, Daniela Parente de Mello \\
\hline $\begin{array}{l}\text { Um modelo de avaliação dos fatores sistêmicos } \\
\text { determinantes do valor e custo de capital de uma empresa } \\
\text { nos países desenvolvidos e nos emergentes / Daniela } \\
\text { Parente de Mello Modiano ; orientador: Luiz Felipe } \\
\text { Jacques da Motta. }-2007 \text {. } \\
\qquad 106 \mathrm{f} \text {; } 30 \mathrm{~cm}\end{array}$ \\
\hline $\begin{array}{l}\text { Dissertação (Mestrado em Administração)- } \\
\text { Pontifícia Universidade Católica do Rio de Janeiro, Rio de } \\
\text { Janeiro, } 2007 \text {. } \\
\text { Inclui bibliografia }\end{array}$ \\
\hline $\begin{array}{l}\text { 1. Administração - Teses. 2. Custo de capital. } 3 . \\
\text { Avaliação de empresa. 4. Competitividade das nações. I. } \\
\text { Motta, Luiz Felipe Jacques da. II. Pontifícia Universidade } \\
\text { Católica do Rio de Janeiro. Departamento de } \\
\text { Administração. II. Título. }\end{array}$ \\
\hline
\end{tabular}

CDD: 658 
Aos meus pais, Eduardo e Isabel, que muito me ajudaram a acreditar que a realização desta dissertação fosse possível.

Ao Filipe, por todo carinho e compreensão, durante os períodos de estudo e de preparação da dissertação. 


\section{Agradecimentos}

Ao meu orientador, Luiz Felipe Jacques da Motta, pelo apoio e parceria na elaboração deste trabalho.

Aos meus colegas de sala de aula da PUC-RIO e de trabalho da BHPBilliton.

Aos funcionários do IAG, pela disposição em ajudar-me a todo o momento, em especial à Teresa e ao Leopoldo.

A todos os amigos e familiares que de uma forma ou de outra me estimularam ou me ajudaram.

Aos professores que participaram da Comissão examinadora. 


\section{Resumo}

Modiano, Daniela; da Motta, Luiz Felipe Jacques. Um modelo de avaliação dos fatores sistêmicos determinantes do valor e custo de capital de uma empresa nos países desenvolvidos e nos emergentes. Rio de Janeiro, 2007. 106p. Dissertação de Mestrado (Opção profissional) - Departamento de Administração, Pontifícia Universidade Católica do Rio de Janeiro.

O presente estudo tem por objetivo entender as variáveis que influenciam de forma positiva ou negativa o custo de capital e também o valor das empresas. As empresas se encontram inseridas em um ambiente que lhes exerce forças externas, sejam elas econômicas, fiscais e financeiras, sociais, políticas e institucionais, legais ou regulatórias, internacionais e tecnológicas. A compreensão dos fatores sistêmicos e o impacto das suas mudanças na empresa tem sido um dos grandes desafios da economia moderna, dando a maior correlação entre a macro e a microeconomia. É também apresentada uma revisão da literatura nacional e internacional sobre a competitividade das nações e as metodologias mais utilizadas para o cálculo do custo de capital e avaliação de uma empresa. Portanto, o estudo foi efetuado com base nos dados anuais de competitividade dos países produzidos pelo IMD (International Institute for Management Development) e os dados de valores de empresas fornecidos pelo MSCI (Morgan Stanley Capital Index). Além disso, optou-se por segregar estes países de acordo com seu estágio de desenvolvimento, ou seja, emergentes e desenvolvidos. Para isso, foram utilizados os métodos estatísticos conhecidos como Regressão Múltipla, assim como uma pesquisa profunda nos artigos sobre este tema. Por fim, os resultados serão expostos e as conclusões apresentadas.

\section{Palavras-chave}

Custo de Capital, Avaliação de Empresa e Competitividade das Nações 


\section{Abstract}

Modiano, Daniela; da Motta, Luiz Felipe Jacques. A determining systemic factor's evaluation model of a company's value and capital cost in the developing and emerging countries. Rio de Janeiro, 2007. 106p. MSc. Dissertation, Departamento de Administração, Pontifícia Universidade Católica do Rio de Janeiro.

The present study has the objective of understand the variables influencing positively or negatively the capital cost and the value of companies. Companies are inserted in an environment that exerts external forces, such as economic, fiscal and financial, social and institutional, legal or regulatory, international and technological. The understanding of systematical factors and the impact of those changes in the company have been one of the great challenges of the modern economy, giving to the biggest correlation between the macro and the micro economy. It is also presented a revision of national and international literature on the competitiveness of the nations and the methodologies more used for the calculation of the capital cost and valuation of assets. Therefore, the study was accomplished with the annual data of The World Competitiveness Yearbook produced by IMD (International Institute for Management Development) and an index provided by MSCI (Morgan Stanley Capital Index) with the market value of companies. The countries were separated by their development stage, such as emerging and developed countries. For this, it was used the statistical method known as Multiple Regression, as well as, some articles on this subject. Finally, the results will be displayed and the conclusions presented.

\section{Palavras-chave}

Cost of Capital, Valuation and Competitiveness of Nations 


\section{Sumário}

1 Introdução 14

1.1. Objetivos da Pesquisa $\quad 15$

1.2. Relevância do Estudo 16

1.3. Delimitação do Estudo 16

$\begin{array}{ll}\text { 1.4. Organização da Pesquisa } & 18\end{array}$

2 Referencial Teórico 19

2.1. Competitividade 19

2.2. Avaliação de Empresas 22

2.2.1. Modelo de Avaliação de Gordon 22

2.2.2. Modelo de Avaliação Relativa ou por Múltiplos 23

2.2.3. Teoria das Opções Reais 24

2.2.4. Modelo de Avaliação por Fluxo de Caixa Descontado 25

2.2.4.1. Modelo de Desconto dos Fluxos de Caixa Líquidos do Acionista (FCFE - Free Cashflow to Equity) 26

2.2.4.2. Modelo de Desconto dos Fluxos de Caixa Líquidos da Empresa (FCFF - Free Cashflow to Firm) 27

2.3. Custo de Capital 29

2.3.1. Custo de capital de terceiros 29

2.3.2. Custo de capital de próprio 29

3 Metodologia 32

3.1. Tipo de Pesquisa 32

3.2. População e Amostra 33

3.3. Metodologia de Classificação dos Dados - Variáveis dependentes 34

3.4. Metodologia de Classificação dos Dados - Variáveis independentes 35

3.5. Métodos estatísticos para Análise dos Dados 37 
4 Análise dos Dados $\quad 40$

4.1. Segmentação 40

4.2. Análise das premissas para regressão linear multivariada 42

4.3. Análise das premissas - Custo de Capital - Países Emergentes 42

4.3.1. Análise de normalidade das variáveis 42

4.3.2. Análise de homogeneidade da variância 44

4.3.3. Análise de linearidade 45

4.3.4. Análise de regressão linear multivariada 48

4.3.5. Análise de regressão linear multivariada (Stepwise) 50

4.4. Análise das premissas - Custo de Capital - Países Desenvolvidos51

4.4.1. Análise de normalidade das variáveis 51

4.4.2. Análise de homogeneidade da variância 53

4.4.3. Análise de linearidade 54

4.4.4. Análise de regressão linear multivariada 55

4.4.5. Análise de regressão linear multivariada (Stepwise) 57

4.5. Análise das premissas - Valor de Empresa - Países Emergentes 58

4.5.1. Análise de normalidade 58

4.5.2. Análise de homogeneidade da variância 59

4.5.3. Análise de linearidade 60

4.5.4. Análise de regressão linear multivariada 62

4.5.5. Análise de regressão linear multivariada (Stepwise) 65

4.6. Análise das premissas - Valor de Empresa - Países

Desenvolvidos 66

4.6.1. Análise de homogeneidade da variância 67

4.6.2. Análise de linearidade 68

4.6.3. Análise de regressão linear multivariada 70

4.6.4. Análise de regressão linear multivariada (Stepwise) 72

4.7. Resumo das Análises $\quad 73$

4.7.1. Custo de Capital $\quad 73$

4.7.1.1. Custo de Capital - Países Emergentes 74

4.7.1.2. Custo de Capital - Países Desenvolvidos 74

4.7.2. Valor das empresas $\quad 74$

4.7.2.1. Valor das empresas - Países Emergentes 74 
4.7.2.2. Valor das empresas - Países Desenvolvidos

5 Conclusões $\quad 75$

5.1. Custo de Capital $\quad 77$

5.1.1. Custo de Capital - Países Emergentes 77

5.1.2. Custo de Capital - Países Desenvolvidos 79

5.2. Valor de Empresa $\quad 80$

5.2.1. Valor de Empresa - Países Emergentes 80

5.2.2. Valor de Empresa - Países Desenvolvidos 82

6 Referências Bibliográficas Consultadas $\quad 84$

7 Anexos $\quad 88$

7.1. Lista dos países - Em ordem alfabética 88

7.2. Árvore Estrutural Com os Fatores, Sub-fatores e Critérios que medem a Competitividade Mundial 90

7.3. Lista de Fatores, Sub-Fatores e Critérios 91

7.4. Índice 104 


\section{Lista de tabelas}

Tabela 1 - Sub-fatores que influenciam o custo de capital 35

Tabela 2 - Subfatores que influenciam o valor das empresas 35

Tabela 3 - Relação dos Países Emergentes e Desenvolvidos 41

Tabela 4 - Teste de Normalidade para países emergentes

(Kolmogorov-Smirnov) 43

Tabela 5 - Teste de Homogeneidade da Variância - Países Emergentes 44

Tabela 6 - Teste de Linearidade para Países Emergentes (Resumo do Modelo) 45

Tabela 7 - Teste de Linearidade para Países Emergentes 47

Tabela 8 - Regressão Linear Multivariada para Países Emergentes 48

Tabela 9 - Regressão Linear Multivariada para Países Emergentes

- Coeficientes 49

Tabela 10 - Resumo do Modelo (Stepwise) - Países Emergentes 50

Tabela 11 - Coeficientes (stepwise) - Países Emergentes 51

Tabela 12 - Teste de Normalidade para países desenvolvidos

(Kolmogorov-Smirnov) 52

Tabela 13 - Teste de Homogeneidade da Variância - Países Desenvolvidos 53

Tabela 14 - Teste de Linearidade para Países Desenvolvidos 54

Tabela 15 - Regressão Linear Multivariada para Países Desenvolvidos 55

Tabela 16 - Regressão Linear Multivariada para Países

Desenvolvidos (coeficientes) 56

Tabela 17 - Resumo do Modelo (Stepwise) - Países Desenvolvidos 57

Tabela 18 - Coeficientes (Stepwise) - Países Desenvolvidos 57

Tabela 19 - Teste de Normalidade para países emergentes

(Kolmogorov-Smirnov) 58

Tabela 20 - Teste de Homogeneidade da Variância - Países Desenvolvidos 59

Tabela 21 - Teste de Linearidade para Países Emergentes 61

Tabela 22 - Regressão Linear Multivariada para Países Emergentes 62

Tabela 23 - Regressão Linear Multivariada para Países

Emergentes (coeficientes) 63

Tabela 24 - Regressão Linear Multivariada para Países Emergentes 65 
Tabela 25 - Regressão Linear Multivariada para Países

Emergentes (coeficientes)

66

Tabela 26 - Teste de Normalidade para Países Desenvolvidos

(Kolmogorov-Smirnov) 66

Tabela 27 - Teste de Homogeneidade da Variância - Países Desenvolvidos 67

Tabela 28 - Teste de Linearidade para Países Desenvolvidos

(Resumo do Modelo) $\quad 68$

Tabela 29 - Teste de Linearidade para Países Desenvolvidos 69

Tabela 30 - Regressão Linear Multivariada para Países Desenvolvidos 70

Tabela 31 - Regressão Linear Multivariada para Países

$\begin{array}{ll}\text { Desenvolvidos (coeficientes) } & 71\end{array}$

Tabela 32 - Regressão Linear Multivariada para Países Desenvolvidos 73

Tabela 33 - Regressão Linear Multivariada para Países

Desenvolvidos (coeficientes) 73 


\section{Lista de figuras}

Figura 1 - Diamante de Porter

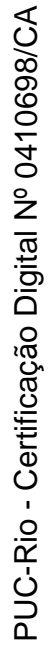

